\title{
Introduction to the Special Issue on EC'15
}

The 16th ACM Conference on Electronic Commerce (EC'15) was held on June 15-19, 2015, in Portland, Oregon, as part of the Federated Computing Research Conference (FCRC'15). EC is the leading scientific conference on advances in theory, systems, and applications at the interface of economics and computation. The conference focuses on research related to the areas of Theory and Foundations, Artificial Intelligence and Applied Game Theory, and Experimental, Empirical, and Applications. The ACM Transactions on Economics and Computation (TEAC) journal is proud to present a special issue containing extended versions of a selected set of papers presented at EC'15. We invited a set of papers that received extremely positive conference reviews; each paper that took us up on this invitation subsequently went through TEAC's own independent and rigorous review process. The end result is a set of 11 stellar articles that will appear in this issue of TEAC:

- David Kurokawa, Ariel D. Procaccia, and Nisarg Shah: Leximin Allocations in the Real World

- Sampath Kannan, Jamie Morgenstern, Ryan Rogers, and Aaron Roth: Private Pareto Optimal Exchang

- Avrim Blum, Yishay Mansour, and Jamie Morgenstern: Learning What's Going on: Reconstructing Preferences and Priorities from Opaque Transactions

- Hossein Esfandiari, Nitish Korula, and Vahab Mirrokni: Online Allocation with Traffic Spikes: Mixing Adversarial and Stochastic Models

- Hoda Heidari, Sebastien Lahaie, David M. Pennock, and Jennifer Wortman Vaughan: Integrating Market Makers, Limit Orders, and Continuous Trade in Prediction Markets

- Joseph (Seffi) Naor and David Wajc: Near-Optimum Online Ad Allocation for Targeted Advertising

- Jon Kleinberg and Maithra Raghu: Team Performance with Test Scores

- Parikshit Gopalan, Noam Nisan, and Tim Roughgarden: Public Projects, Boolean Functions, and the Borders of Border's Theorem

- Aviad Rubinstein and S. Matthew Weinberg: Simple Mechanisms for a Subadditive Buyer and Applications to Revenue Monotonicity

-Constantinos Daskalakis, Nikhil R. Devanur, and S. Matthew Weinberg: Revenue Maximization and Ex-Post Budget Constraints

- Slava Bronfman, Noga Alon, Avinatan Hassidim, and Assaf Romm: Redesigning the Israeli Medical Internship Match

This collection of papers highlights the connections between theory and practice that is a hallmark of EC. It includes applications to Internet advertising, medical internship matching, team-building, and prediction markets. The growing interest in privacy considerations is reflected, as are new developments in the theory of maximizing revenue and learning preferences in auctions for multiple goods.

2018 Copyright is held by the owner/author(s).

2167-8375/2018/10-ART10

https://doi.org/10.1145/3274531 
We thank all of the authors who submitted articles to this special issue and worked hard on extending and crystallizing their papers. We also thank all of the reviewers whose feedback both ensured the quality of this special issue and in many cases helped to improve the articles themselves. We hope that you enjoy reading these articles and that they inspire more great research.

Michal Feldman

Tel Aviv University

Associate Editor

Brendan Lucier

Microsoft Research

Michael Schwarz

Google

Guest Editors 